\title{
An Introduction to this Special Issue: Animal Models of Human Chemosensory Perception and Behavior
}

\author{
Christopher T. Simons ${ }^{1}$
}

Received: 21 July 2015 / Accepted: 24 July 2015 / Published online: 7 August 2015

(C) Springer Science+Business Media New York 2015

In this Special Issue of Chemosensory Perception, the important link connecting chemosensory work in animals to human perception and behavior is highlighted. Historically, the use of animal models has been instrumental in developing our understanding of the human condition. Tremendous insight and scientific advancement has been made through the development of applicable animal models. In the case of the chemical senses, this has been especially true as animal models have been used to identify the key proteins involved in chemosensory transduction; to investigate the neural code underpinning taste, smell, and chemesthetic sensations; and, more recently, to examine the function of taste and smell receptors in roles other than gustation and olfaction.

Linking results from animal and human studies is critically important to the chemical senses discipline. For those scientists whose techniques and interests focus primarily on human perception, a clear understanding of the molecular and physiological phenomena underpinning chemosensation provides a fundamental platform upon which to base further hypotheses. On the other hand, the impact of animal work is greatest when findings are translated to human significance. Moreover, the chemosensory community is unique in that the largest global industry - food, beverage, and consumer products - is a direct beneficiary of the research efforts and can leverage results to improve the quality, healthiness, and acceptance of

Christopher T. Simons

simons.103@osu.edu

1 Department of Food Science and Technology, The Ohio State University, Columbus, OH, USA products in this category. As such, translatable findings help fuel the economic engine of this important industrial sector.

In the current Special Issue of Chemosensory Perception, six papers have been authored with the intent to provide human relevance to chemosensory studies completed using animals. The articles represent a small collection of the numerous animal studies that have contributed to our current knowledge, yet provide broad perspective covering topics of interest within chemosensation. Three of the articles focus on the sense of taste and provide evidence that data obtained in rodents are relevant to understanding appetitive taste processing and ingestive behavior, as well as offering key insights related to human issues of obesity and weight management. The fourth article concerns olfaction and posits an interesting role of the lung in retronasal odor perception. The last two articles focus on chemesthesis, particularly the remarkable overlap of trigeminal chemosensitivity obtained from animals and human psychophysical work as well as the role of thermosensitive transient receptor potential (TRP) channels in touch, temperature, and pain sensation.

I would like to thank the authors for contributing these papers and acknowledge their time and dedication to preparing these manuscripts. As an advocate of linking human perception and behavior to underlying neurobiological mechanisms, I believe the content of this Special Issue of Chemosensory Perception makes an important contribution to the field of chemosensation. I hope you agree.

Happy Reading!

Christopher T. Simons

\section{Compliance with Ethics Requirements}

Conflict of Interest Christopher T. Simons declares that he has no conflict of interest.

This article does not contain any studies with human participants or animals performed by any of the authors. 